\title{
Sleep physiology and sleep disorders in childhood
}

This article was published in the following Dove Press journal:

Nature and Science of Sleep

3 September 201 I

Number of times this article has been viewed

\section{Hanan M El Shakankiry \\ King Fahd University Hospital, Al Dammam University, Al Khobar, Kingdom of Saudi Arabia}

Correspondence: Hanan M El Shakankiry King Fahd University Hospital, Pediatric Neurology Division, Pediatric Department, PO Box 40284, Al Khobar 31952, Kingdom of Saudi Arabia Tel +96 6558404028

Fax +96 638966709

Email hshakankiry@hotmail.com
Abstract: Sleep has long been considered as a passive phenomenon, but it is now clear that it is a period of intense brain activity involving higher cortical functions. Overall, sleep affects every aspect of a child's development, particularly higher cognitive functions. Sleep concerns are ranked as the fifth leading concern of parents. Close to one third of all children suffer from sleep disorders, the prevalence of which is increased in certain pediatric populations, such as children with special needs, children with psychiatric or medical diagnoses and children with autism or pervasive developmental disorders. The paper reviews sleep physiology and the impact, classification, and management of sleep disorders in the pediatric age group.

Keywords: sleep physiology, sleep disorders, childhood, epilepsy

\section{Phenomenon of sleep}

Sleep is a cyclic, physiologic state of decreased responsiveness from which individuals arise spontaneously. ${ }^{1}$ It has long been considered as a largely negative phenomenon in which vigilance is switched off. However, it is now clear that it is a period of intense brain activity involving higher cortical functions with considerable physiologic activity, to the extent that the brain is considered more active during sleep than during wakefulness. ${ }^{2-4}$

Infants and young children spend a majority of their time asleep, suggesting that sleep is important for the developing brain and body. By 3 years of age, the child has spent more time sleeping than in all wakeful activities combined. ${ }^{3}$

The importance of sleep to overall health and well-being is becoming increasingly appreciated, and the lack of adequate education in the area of sleep is a concern that has only recently gained recognition. ${ }^{5}$ Sleep-wake disorders afflict close to a third of all children. ${ }^{1}$ Approximately $25 \%$ of children younger than 5 years experience some type of sleep problem. ${ }^{6}$

\section{Physiology}

Familiarity with sleep physiology is fundamental to understanding pediatric sleep disorders. ${ }^{1}$ Sleep occurs in reptiles and birds, and nearly all mammals sleep and dream. Sleep is necessary for life, with sleep deprivation in the rat being reported to result in weight loss despite increased food intake, metabolic and thermoregulatory imbalance, and death in about 1 month. ${ }^{7}$

The two main neurobiologic processes believed to regulate sleep and wakefulness are the circadian process, an internal clock that dictates periods of wakefulness and sleep based on a light-dark cycle, and the homeostatic process, in which the requirement for sleep builds up during waking hours and is relieved by sleep. ${ }^{8,9}$ 
The circadian rhythm is actually about 25 hours in the absence of synchronization with cues from the environment, thus longer than the 24-hour clock day. Light exposure signals waking and darkness signals sleep, therefore, light exposure prior to the onset of sleep can interfere with sleep onset. Many other social and environmental cues affect the circadian rhythm, including mealtimes, ambient temperature, noise, bedtime routines, physical activity, pain, and medications. ${ }^{4}$ There is also a circadian rhythm to other functions, including endocrine hormone secretion, core body temperature, and sensory processing. ${ }^{10}$ Sleep-wake rhythms are closely linked to body temperature, ie, one tends to be most sleepy around the nadir of core body temperature, which is 4 am, whereas a rise in body temperature during the morning coincides with alertness. ${ }^{1}$

The homeostatic process is the mechanism that involves the sleep debt accumulated during waking hours, which leads to an increase in the sleep drive. It enables the body and mind to rejuvenate and restores alertness. ${ }^{9}$

During sleep, an ultradian rhythm determines the timing and duration of sleep states. The ultradian rhythm refers to the alternation of two distinct types of sleep, ie, nonrapid eye movement (NREM) and rapid eye movement (REM) sleep throughout the sleep period. Each type is associated with distinctive levels of arousal, autonomic response, brain activity, and muscle tone. ${ }^{5}$

NREM sleep has been categorized into four distinct stages according to electroencephalographic changes. The stages represent gradations in depth of sleep and difficulty of arousal, with stage 1 being the lightest and stage 4 being the deepest. ${ }^{4}$ The differentiation of NREM sleep into four distinct stages is completed by 6 months of age. The distinguishing electroencephalographic characteristics of the NREM stages are:

- Stage I or drowsiness, mixed frequency (theta, alpha, and delta), isolated vertex sharp transients, slow rolling eye movements

- Stage II, prominent sleep spindles and K complexes

- Stage III, delta slowing constitutes $20 \%-50 \%$ of a 30 second epoch

- Stage IV, delta slowing constitutes $50 \%$ or more of a 30 second epoch. ${ }^{1}$

When first drifting off, the child enters stage 1 NREM sleep, which is characterized by reduced body movements and responsiveness. This stage comprises approximately $2 \%-5 \%$ of total sleep, with the majority occurring at the beginning of the sleep period. Stage 2 sleep then follows, and is considered the onset of true sleep. It is characterized by decreased eye movements, reduced muscle tone, and deceleration of respiration and heart rate. The child may move freely and reposition in bed. About one half of the total sleep time is spent in stage 2, with the majority occurring in the middle of the night. The third and fourth stages are nearly identical and are together called delta, deep, or slow-wave sleep. These stages are characterized by a relaxed body position, slow and rhythmic breathing, and a decreased heart rate. Arousal is difficult and if awakened, the child will appear confused and disoriented. The predominance of slow-wave sleep is seen in the early hours of the sleep period, and constitutes approximately $20 \%$ of total sleep time. ${ }^{4,5,18}$

REM sleep is characterized by bursts of rapid eye movements, electroencephalographic activity similar to that of the awake state, muscle paralysis, and vividly remembered dreams. Muscle twitches, facial expressions, and vocalizations related to the dream are observed, and frequent changes in respiration and heart rate are also common. ${ }^{11}$ The muscle paralysis in the presence of intense brain activity has led to the synonymous term "paradoxical sleep," whereby the paralysis protects the child from physically acting out the action in dreams. ${ }^{4}$ This stage is considered the time during which the brain "learns" from the experiences of the day. The proportion of REM sleep is highest in infancy (55\%) and declines to about $20 \%-25 \%$ by the age of 5 years. ${ }^{11,12}$

\section{Normal sleep development In the newborn}

At birth, the circadian rhythm is not fully developed, so sleep can occur as easily during the daytime hours as during the night. The normal, full-term newborn sleeps approximately 16-18 hours per day. The pattern of sleep and wakefulness is irregular, with the longest continuous sleep period being 2.5-4.0 hours, often synchronized with feeding times. ${ }^{12}$

Three types of sleep are recognized in the newborn, ie, quiet sleep (equivalent to NREM sleep), active sleep (equivalent to REM), and indeterminate sleep. Quiet sleep is characterized by minimal muscle movements and rhythmic breathing cycles. During active sleep, sucking motions, twitches, smiles, frowns, irregular breathing, and gross limb movements (contrary to the typical REM sleep paralysis seen at later ages) are seen. ${ }^{13}$

In the first few weeks of life, a sleep cycle consists of both active and quiet sleep periods in equal proportion. Each cycle lasts 50-60 minutes and sleep onset occurs through active (REM) sleep. ${ }^{14}$ Each sleep period lasts only one or two sleep cycles. Sleep periods are therefore shorter and more frequent than in an older child, resulting in easily interrupted sleep. ${ }^{5}$ 


\section{In infants and children}

Infants younger than 6 months spend $50 \%$ of their sleep time in REM sleep, compared with $20 \%$ in adults. In infants, sleep starts with an initial active REM stage, in contrast with adults, who do not commonly enter REM sleep until 90 minutes into the sleep cycle. Until 6 months of age, sleep cannot be subdivided into the four electroencephalographic stages seen in the mature sleep pattern. By 6 months, the infant's sleep architecture closely resembles that of an adult's. The infant normally drifts from stage 1 NREM sleep into stage 3 or 4, may return to stage 1 and cycle again. After one to two cycles of NREM sleep, the infant enters REM stage. The first one third of the night is spent mostly in deep sleep (NREM stages 3 and 4) and the last one half is predominantly stage 2 NREM and REM (Figure 1). ${ }^{12,15}$

Night-time sleep gradually becomes consolidated over the first year into a single continuous block, and daytime sleep gradually decreases over the first 3 years. By the age of 4 years, most children no longer require a daytime nap. Night-time sleep needs gradually decrease to become similar to the needs of an adult by adolescence. ${ }^{15}$

\section{Scope of sleep problems Prevalence}

The overall prevalence of a variety of parent-reported sleep problems have been estimated to range from $25 \%$ to $50 \%$ in preschool-aged samples to $37 \%$ in a community sample of $4-10$ year-olds. ${ }^{16}$ More than $40 \%$ of adolescents were also reported to have significant sleep complaints. ${ }^{17}$ A number of studies suggested that adolescents get less than adequate sleep on a regular basis. ${ }^{18,19}$ Sleep problems are more prevalent in certain pediatric populations, such as children with special needs, and in children with psychiatric or medical disorders. The prevalence of severe sleep problems in children with autism or pervasive developmental delays is estimated to be more than $40 \% .{ }^{20}$ Moreover, sleep concerns were ranked as the fifth leading concern of parents, following illness, feeding, behavioral problems, and physical abnormalities. Parents were also noted to be more concerned about sleep problems than difficulties with language development, motor development, toileting, and teething. ${ }^{21}$

\section{Impact}

Sleep problems in children and adolescents have a significant impact on all aspects of functioning. ${ }^{22}$ A wealth of empiric evidence indicates that children and adolescents experience significant daytime sleepiness as a result of inadequate sleep. However, inadequate sleep in children may not manifest as drowsiness, yawning, and the "classic" manifestations of sleepiness that occur in adults. Instead, sleepiness often takes the form of mood and behavioral disturbances such as hyperactivity, poor impulse control, and neurocognitive dysfunctions, such as inattention, which result in significant social and learning problems. ${ }^{21,23}$ Higher-level cognitive functions, such as cognitive flexibility and the ability to reason and think abstractly, and other neuropsychologic functions, such as attention and memory, are also affected by disturbed sleep. In addition, health outcomes of inadequate sleep include an increase in accidental injuries, as well as deleterious effects on the cardiovascular, immune, and various metabolic systems, including glucose metabolism and endocrine function. ${ }^{24,25}$ Moreover, sleep problems are a significant cause of distress for parents, and may be

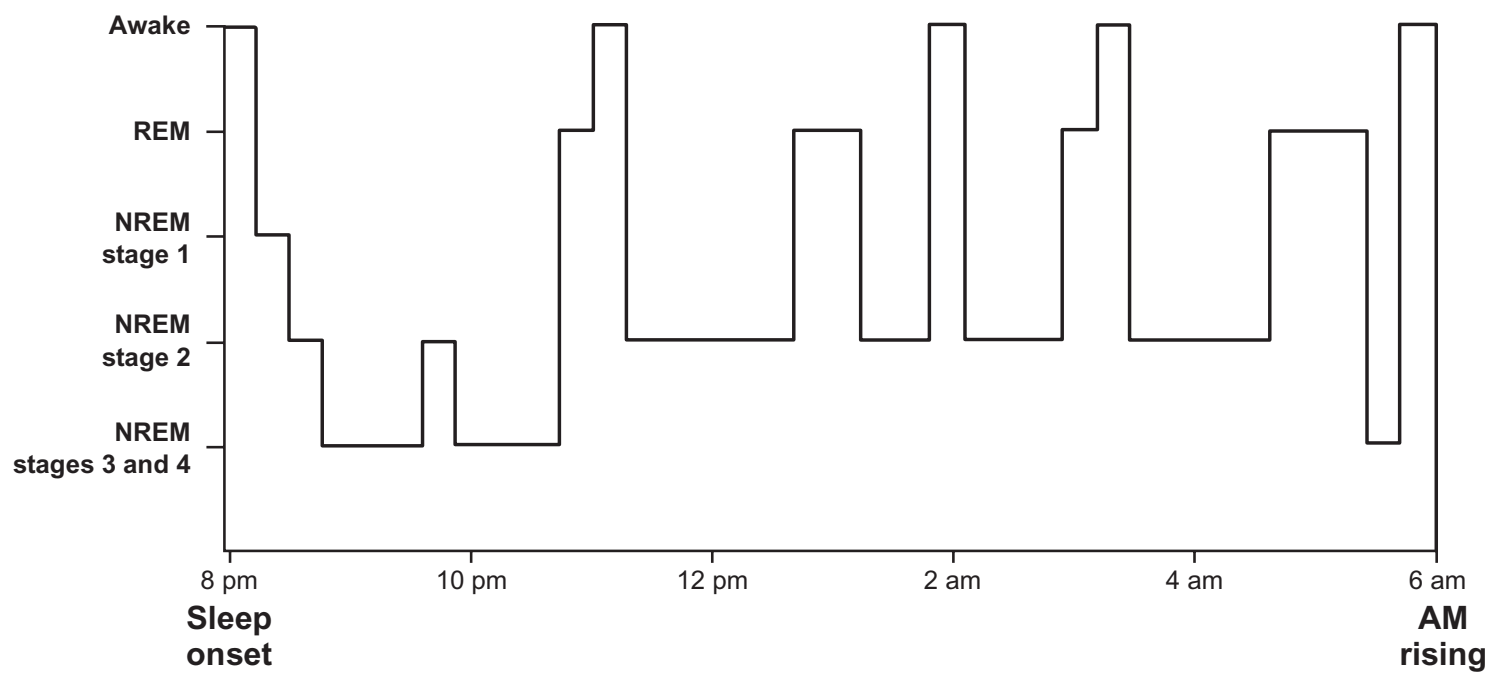

Figure I Schema of typical night sleep pattern of sleep states and stages.

Abbreviations: REM, rapid eye movement; NREM, nonrapid eye movement.

Note: Reprinted from: Adair RH, Bauchner H. Sleep problems in childhood. Current Problems in Pediatrics. 1993;23(4):147-I70 with permission from Elsevier. 
one of the primary reasons for caregiver stress in families with children who have chronic illnesses. ${ }^{26,27}$

\section{Assessment of the patient Sleep history}

Evaluation of sleep problems involves a thorough history of the child's 24-hour routine, focusing on bedtime habits, night-time behavior, naps, and daytime behavior. ${ }^{6}$ Questions relevant to infants and preschool-aged children include the sleep environment (eg, crib or parents' room), sleep position (eg, prone or supine), need for sleep aids (eg, pacifier, rocking, patting), the time a child goes to bed and time of the final morning awakening, presence of habitual snoring, mouth breathing, sweating, gastroesophageal reflux, abnormal behavior suggestive of seizures and parasomnia, behavior during the daytime (irritability, hyperactivity, sleepiness), number of daytime naps and their duration, medication that may affect sleep-wake function, and the interventions the parents carry out to improve sleep. In school-aged children, inquiries regarding sleep environment and the sleep position are to be replaced by questions about the events that take place in the 2-3 hours prior to bedtime (eg, exercise, heavy meals) and daytime assessment should include questions about involuntary naps in the classroom, cataplexy, hypnagogic hallucinations, and any medication tried to promote alertness. $^{1}$

A sleep diary can be helpful in delineating the exact sleep pattern, and in collecting information regarding bedtime, frequency and duration of night-time wakings, nap frequency and duration, time of morning waking, and total sleep time. ${ }^{28}$ Data should be collected for a minimum of 2 weeks, including weekends, to reflect an accurate picture of the sleep problem. ${ }^{6}$

During the evaluation of any sleep problem, cultural differences are important to consider because definitions of bedtime resistance and night waking are, to a large extent, culturally determined. Families must be evaluated in light of their own cultural views. There is a widely held belief that all infants should sleep alone and achieve the developmental milestone of "sleeping through the night," and that cosleeping may impair the child's ability to develop independence. ${ }^{29}$ However, in many cultures, cosleeping is common practice. ${ }^{30}$ Breastfeeding families also tend to have high rates of cosleeping. Research on cosleeping has shown mixed results, with some studies reporting that children experience decreased slow wave sleep and more frequent night awakenings, whereas other studies do not support these findings. ${ }^{6,31}$

\section{Physical examination}

A thorough physical examination should be performed in any child with a sleep complaint to look for medical causes that may disrupt sleep, such as otitis media (pain), chronic rhinitis (airway obstruction), and eczema (nocturnal itching). Chronic disorders, such as asthma, cerebral palsy, and blindness, can also result in sleep problems. ${ }^{12}$ Physical examination should focus on the causes and consequences of sleep-related disorders, and whether there are signs of failure to thrive, obesity, congenital anomalies of the nervous system or airway, abnormal facies, mouth breathing, enlarged tonsils or adenoids, bulbar dysfunction, clubbing, cyanosis, edema, or a heart murmur. ${ }^{32}$

\section{Investigations}

Polysomnography, the technique of monitoring multiple physiologic parameters during sleep, is useful in the evaluation of intrinsic sleep disorders, and can also help to differentiate sleep disorders from nocturnal seizures. ${ }^{28}$ Generally, the electroencephalogram, eye movements, chin electromyogram, nasal airflow, thoracic and abdominal respiratory effort, electrocardiogram, and oxygen saturation are recorded continuously during sleep on a computerized system or on paper. Standard criteria for the scoring of sleep stages and respiratory events have been established. ${ }^{1}$

The Multiple Sleep Latency Test (MSLT), on the other hand, consists of five naps that are recorded throughout the day at 2-hour intervals. Sleep latency (time taken to fall asleep) and REM latency (time until REM onset) are recorded for each nap. Normative values have been established for mean sleep latency, which is reported to range from 12 to 18 minutes, and is noted to decrease with increases in the Tanner stage of sexual development. Pathologic sleepiness is defined as an average sleep latency of less than 8 minutes. Normal alertness in preadolescent children is associated with average sleep latencies longer than 15 minutes. To determine whether daytime sleepiness is a consequence of abnormal night sleep, the test must be preceded the night before by polysomnography. ${ }^{1,32}$

Actigraphy, that involves the continuous recording and storing of skeletal muscle activity for 1-2 weeks in a wristwatch-like microcomputer device, equates periods of muscle activity with wakefulness and lack of muscle activity with sleep. There is generally good correlation between the polysomnogram and actigraph-defined total sleep time, sleep latency, and sleep efficiency (percentage of time in bed spent sleeping). ${ }^{1}$ 
Other diagnostic studies are also indicated based on history, physical examination, and sleep studies, including: soft tissue films of the airway if there is obstruction; echocardiogram and electrocardiogram in cases associated with heart failure; magnetic resonance imaging of the posterior fossa in patients with central apnea or other neurologic problems associated with control of breathing, signs of increased intracranial pressure, or diseases associated with brainstem compression; $\mathrm{pH}$ probe and upper gastrointestinal films to evaluate reflux; pulmonary function studies to assess breathing; and endocrine studies, especially thyroid function, should be obtained in patients with obesity or with Down syndrome. $^{32}$

\section{Classification of sleep disorders}

There are more than 80 ICD-9 classified sleep disorders, and scores of causes for both sleepiness and insomnia. The International Classification of Sleep Disorders was originally published in 1990 and was subsequently modified. ${ }^{33,34}$ Each sleep disorder must be considered in the context of age, because age influences the presentation; for example, sleep-disordered breathing resulting in adult sleepiness can contribute to death in infants. Moreover, a vast variety of intrinsic and extrinsic factors influence the presentation of sleep disorders in children, including stage of maturity of the nervous system, compliance of the chest wall, and obesity. ${ }^{35}$

Dyssomnias are problems in which the child has trouble either falling or staying asleep at night, or experiences excessive sleepiness during the day. ${ }^{28}$ Dyssomnias are the major cause of disturbed night-time sleep and daytime sleepiness. ${ }^{35}$ They are noted in Diagnostic and Statistical Manual of Mental Disorders Fourth Edition ${ }^{36}$ as disorders of initiating or maintaining sleep, or of excessive sleepiness, which result in a disturbance in the quality, amount, or timing of sleep.

Parasomnias, on the other hand, involve behaviors or physiologic events that interrupt sleep after sleep onset, and include disorders of arousal, partial arousal, or with transition between the stages of sleep, ${ }^{28}$ ie, activation of the physiologic systems at inappropriate times during the sleep cycle. ${ }^{5}$ Although the exact prevalence is unknown, as a group, they are frequently experienced at the age of 3-8 years, ${ }^{3}$ and children who experience one of these problems are likely to experience symptoms of another sleep problem. ${ }^{6,14}$ Table 1 lists the different sleep disorders based on The International Classification of Sleep Disorders, Revised 2001. ${ }^{34}$

\section{Intrinsic dyssomnias}

These include disorders that arise from an abnormality of the patient that alters the sleep process, and may include both psychologic and organic problems, such as obesity, congenital anomalies, obstructive sleep apnea, or primary abnormalities of sleep physiology, as seen in narcolepsy. ${ }^{32,34}$

\section{Obstructive sleep apnea}

Obstructive sleep apnea comprises the most common form of breathing disorder during sleep. ${ }^{37-39}$ Normally, breathing during sleep is quiescent, but intermittent periods of snoring can be noted. In contrast to intermittent snoring, chronic loud snoring may be a sign of at least partial, intermittent airway obstruction. Apnea events during sleep cause acute hemodynamic alterations that can result in systemic and pulmonary hypertension, decreased cardiac output, and increased right and left afterload. ${ }^{40}$ In the obstructive type, airflow is diminished or absent in the presence of continued chest and abdominal muscle movement due to partial or complete blockage in the upper airway. This differs from central apnea in which absence of chest and abdominal wall movement is noted, along with an absence of airflow and no apparent upper airway blockage. ${ }^{41}$

Obstructive sleep apnea often results from adenotonsillar hypertrophy, neuromuscular disease, eg, muscular and myotonic dystrophy, congenital myopathies, and spinal muscular atrophy, and craniofacial abnormalities. ${ }^{15,32,42}$ Children with syndromes that involve micrognathia, such as Treacher Collins syndrome, Pierre Robin syndrome, and Goldenhar's syndrome, become obstructed at the hypopharyngeal level. In children with trisomy 21 , a narrow upper airway combined with macroglossia and hypotonic musculature predisposes them to obstructive sleep apnea. ${ }^{1,42}$

The mildest form of obstructive sleep apnea in children is upper airway resistance syndrome. Patients have symptoms of obstructive sleep apnea but lack the accompanying polysomnographic findings. While many children demonstrate intermittent snoring and mouth breathing, true obstructive sleep apnea results in detrimental clinical sequelae, such as failure to thrive, behavior problems, enuresis, and in severe cases cor pulmonale. The presenting problem in children with sleep-disordered breathing depends on the age. In children younger than 5 years, snoring is the most common complaint. Other symptoms reported by parents include mouth breathing, diaphoresis, paradoxic chest movement, restlessness, frequent awakenings, and witnessed apneic episodes. Children 5 years and older commonly present with enuresis, behavioral problems, decreased attention span, and 
Table I Sleep disorders
I. Dyssomnias
a. Intrinsic sleep disorders, eg, sleep disordered breathing, narcolepsy.
b. Extrinsic sleep disorders, eg, sleep-onset association disorder, inadequate sleep hygiene.
c. Circadian rhythm sleep disorders, eg, delayed sleep phase syndrome, irregular sleep-wake pattern.

\section{Parasomnias}

a. Arousal disorders, eg, somnambulism, sleep terror.

b. Sleep-wake transition disorders, eg, sleep starts, sleep talking.

c. Parasomnias usually associated with REM sleep, eg, nightmares, sleep paralysis.

d. Other parasomnias, eg, sleep bruxism.

3. Sleep disorders associated with mental, neurologic, or other medical disorders

a. Associated with mental disorders, eg, psychoses, mood disorders.

b. Associated with neurologic disorders, eg, sleep-related epilepsy.

c. Associated with other medical disorders, eg, sleep-related asthma, sleep-related gastroesophageal reflux.

4. Proposed sleep disorders, eg, short sleeper, long sleeper, terrifying hypnagogic hallucinations, sleep-related neurogenic tachypnea, sleep-related laryngospasm.

Abbreviation: REM, rapid eye movement.

Note: Based on The International Classification of Sleep Disorders, Revised: Diagnostic and Coding Manual, ${ }^{34}$ used with permission of the publisher.

failure to thrive, in addition to snoring. Compared with adults, fewer children with obstructive sleep apnea report excessive daytime somnolence, with the exception of obese children. In extreme cases of obstructive sleep apnea in children, cor pulmonale and pulmonary hypertension may be the presenting problems. ${ }^{40,43-45}$

The hypothesized etiology for failure to thrive is increased work of breathing, with subsequent increase in baseline caloric expenditure. Decreased production of growth hormone during fragmented sleep may contribute further to poor growth. ${ }^{42,46}$ The nocturnal polyuria of obstructive sleep apnea is evoked by the negative intrathoracic pressure due to the inspiratory effort posed against a closed airway, leading to cardiac distension and subsequently the release of atrial natriuretic peptide. This hormone increases sodium and water excretion and inhibits other hormone systems that regulate fluid volume, vasopressin, and the rennin-angiotensinaldosterone complex. Treatment of obstructive sleep apnea has been reported to reverse nocturnal polyuria and thereby reduce or eliminate nocturia and enuresis. ${ }^{47}$

Obstructive sleep apnea in adults is defined as a respiratory pause lasting 10 seconds or more. Children, on the other hand, have less functional residual capacity, faster resting respiratory rates and a more compliant chest wall, so a respiratory pause of 3-4 seconds can lead to desaturation. These findings have led to the development of separate guidelines for the interpretation of polysomnograms in children. ${ }^{42,48}$ The apnea-hypopnea index (AHI) is the total number of apneas (cessation of air flow as shown by the absence of oronasal air flow) and hypopneas (reduction in air flow, seen on either the thermistor or nasal pressure monitor, of greater than $50 \%$ with a desaturation of at least 3\%) per hour of sleep, ie, the number of events of apneas and hypopneas that occur divided by the total duration of sleep. ${ }^{39}$ The normative data for children are not well defined; apnea indices are believed to vary across age groups, and many studies intended better delineation of the normative data in infancy and childhood. ${ }^{39,48}$ However, it is accepted that $\mathrm{AHI} \leq 1$ is considered to be normal and AHI $>1$ is considered diagnostic for obstructive sleep apnea according to pediatric standards. ${ }^{42,48}$

Several options are available for the medical management of obstructive sleep apnea in children. If snoring and obstructive sleep apnea occur intermittently and are associated with recurrent tonsillitis or adenoiditis, antibiotic therapy may help. Recurrent infections accompanied by sleep disturbance may necessitate adenotonsillectomy. Adenotonsillectomy remains the treatment of choice for most children with a strong clinical history of obstructive sleep apnea or with obstructive sleep apnea documented by polysomnography, and numerous studies have documented improvement in snoring, obstructive sleep apnea, enuresis, behavior, and growth following adenotonsillectomy ${ }^{47}$ Continuous positive airway pressure is the treatment of choice when adenotonsillectomy is contraindicated or has failed; adjustment of the mask size every 6 months is recommended. ${ }^{42,49-51}$ In obese children, weight loss is an imperative therapeutic measure. Treatment of rhinitis is important in children with obstructive sleep apnea secondary to nasal obstruction. Nasal steroid use may have a role but is unlikely to provide definitive therapy. ${ }^{51-53}$

Pediatric experience with uvulopalatopharyngoplasty has been limited to children with muscular hypotonia and oropharyngeal tissue redundancy. ${ }^{54}$ Tracheotomy is reserved for use in children with severe obstructive sleep apnea who 
have failed to improve with other medical and surgical treatments and in special cases in which these modalities are contraindicated or unlikely to be of benefit, as in Pierre Robin syndrome. In children with midfacial hypoplasia, craniofacial advancement may be indicated. Glossopexy, mandibular distraction or advancement, or tongue suspension should be considered in patients with micrognathia. ${ }^{55}$

\section{Central alveolar hypoventilation}

Central alveolar hypoventilation, on the other hand, is defective automatic regulation of breathing, which leads to a failure to initiate respiration during sleep. The respiratory pattern is similar to a normal fetal breathing pattern, and it was therefore understood as a failure to switch to mature respiratory patterns at birth. The disorder may present during infancy (congenital form, ie, “Ondine's curse") or in later childhood. The congenital form may be caused by developmental malformations of the brainstem, which may only be apparent on autopsy, and inborn errors of metabolism. Head injury, bulbar poliomyelitis, syringobulbia, and type II Arnold-Chiari malformation, are other underlying disorders, but sometimes no definite etiology is found. In the early stages of this disorder, the respiratory rate and depth are normal during wakefulness, but hypoventilation, hypercarbia, and oxygen desaturation appear during sleep. Challenging breathing, during NREM sleep, using 5\% inhaled carbon dioxide, fails to induce the expected 3-5-fold increase in minute volume. There is no definite satisfactory treatment, but acetazolamide and theophylline are believed to enhance the chemoreceptivity of the brainstem respiratory neurons. Home ventilation via a tracheostomy and diaphragmatic pacing are other treatment modalities. The disorder may prove to be fatal. ${ }^{1,32}$

\section{Narcolepsy}

Narcolepsy is a chronic, lifelong disorder characterized by irresistible attacks of daytime sleepiness lasting 15-30 minutes, cataplexy (sudden loss of muscle tone in association with emotional stimuli like fright or surprise), and sleep paralysis (an inability to move for a few seconds at sleep onset). ${ }^{1}$ In children, it may present merely with excessive daytime sleepiness, without demonstrating cataplexy or other symptoms until after adolescence. ${ }^{56}$ Therefore, it is not uncommon for a child to be symptomatic for several years before the diagnosis of narcolepsy is considered. ${ }^{57,58}$

Narcolepsy shows a genetic predisposition, with close to $100 \%$ of patients possessing the HLA DRB10602 antigen, the prevalence of which is only $25 \%-30 \%$ in the general population. Interestingly, both canine narcolepsy and human narcolepsy are associated with mutations in the hypocretin 2 receptor genes, with a resultant deficiency state. ${ }^{1}$

Cerebrospinal fluid for hypocretin levels, HLA typing, and MSLT can aid in the diagnosis. ${ }^{7,59}$ The diagnostic features of narcolepsy on the nocturnal polysomnogram include a short REM latency of 45-60 minutes and increased sleep fragmentation. On the daytime MSLT, narcoleptics show a very short mean sleep latency of 5 minutes or less (normal 12-18 minutes), with sleep-onset REM periods on two or more nap opportunities of the MSLT. ${ }^{1}$

Daytime sleepiness associated with narcolepsy is treated using stimulants, such as methylphenidate or dextroamphetamine. Cataplexy responds to anticholinergic agents. Sodium oxybate (also known as gamma hydroxybutyrate), which was approved by the US Food and Drug Administration in 2002, is approved specifically for the treatment of cataplexy; it increases the amount of slow wave sleep and improves sleep continuity. Its probable mechanism of action was reported to involve agonist activity at the $\gamma$-aminobutyric acid $\mathrm{B}$ receptor. Improving the quality of nocturnal sleep appears to reduce the severity of all of the classic narcolepsy symptoms. ${ }^{1,60-62}$

\section{Kleine-Levin syndrome}

The rare Kleine-Levin syndrome typically presents in adolescents. There is a predominance of males, with a mean age of onset of 15 years. The episodes of severe hypersomnia and mood disturbance are present in all patients, but variability was observed in the type of mood change, between aggressiveness to decreased psychomotor activity. Hyperphagia and sexual disinhibition are reported in about $50 \%$ of cases, so are therefore not considered necessary to meet the criteria for diagnosis. An association with HLA DQB1*0201 was noted. ${ }^{63}$

\section{Extrinsic dyssomnias Sleep onset association disorder}

Sleep onset association disorder mainly results from poor sleep habits. Night wakings in the infant and preschool-aged child are a normal event; ${ }^{3}$ normal children who are "good sleepers" differ from those who are "poor sleepers" in the ability to self-soothe and return to sleep after awakening. Children learn to expect the conditions that are present at sleep onset and become dependent on them to return to sleep after a night waking. Children who are used to falling asleep in their parents' arms while being rocked or fed a bottle often need their parents to re-establish these conditions after a night waking and are unintentionally trained by their parents to expect these conditions. Parents also experience stress and 
a profound sleep deficit that may result in negative impact on overall family functioning. ${ }^{6,64}$ Management consists of firmly eliminating association with external objects/actions, placing the infant to sleep in his/her own crib, and encouraging the parent to leave the room before the infant falls asleep. Eliminating the daytime naps and postponing bedtime by an hour were also advised as they may make the child sleepier and more prone to fall asleep. ${ }^{1}$

\section{Limit-setting sleep disorder}

Limit-setting sleep disorder is another disorder the most typical presentation of which is preschoolers making excessive demands at bedtime to delay sleep onset. Repeated requests for stories, water, or another television show are common, and bedtime is frequently inconsistent. There is delayed sleep onset due to bedtime resistance, which is defined as requiring more than 20 minutes to fall asleep after going to bed. ${ }^{16}$ The degree of sleep loss depends on the length of time the child requires to fall asleep but is often enough to result in behavior and learning problems during the day. Management includes appropriate bedtime routines, and a consistent bedtime. Parents should be encouraged to be firm in their limit setting, both day and night. ${ }^{6,65}$

\section{Inadequate sleep hygiene}

Inadequate sleep hygiene includes those habits that enhance wakefulness and interrupt the sleep period, leading to a decrease in the quality or quantity of sleep and excessive daytime sleepiness. Examples of these habits include engaging in stimulating activities near bedtime, using the bed for activities not related to sleep (eg, playing, watching television), consumption of caffeine near bedtime, sleeping in uncomfortable bedroom (eg, too cold, hot, bright, or noisy). ${ }^{34,35}$ Inconsistent bedtimes and inappropriate napping are also a major contributor to the problem. ${ }^{66}$ Conversely, appropriate sleep hygiene includes those habits and activities that promote the initiation and maintenance of effective sleep. Therefore, the advised solution is modification of the sleeping environment to promote restful sleep, and parents may also adopt supportive bedtime routines as storytelling. ${ }^{6,32}$

\section{Insufficient sleep syndrome}

Insufficient sleep syndrome is the most common cause of excessive daytime sleepiness in children and adolescents. ${ }^{32}$ It arises when the child fails to get an adequate amount of sleep to maintain appropriate wakefulness during the day. The child's usual sleep period is shorter than expected for age, and the child may experience daytime sleepiness or behaviors indicative of inadequate sleep (eg, inattention, irritability, and hyperactivity). ${ }^{35}$ Adequate or optimal sleep in children is not well defined in the literature, ${ }^{67}$ and the total amount of sleep necessary for peak functioning varies from individual to individual. ${ }^{3}$ It is reported that the child is getting enough sleep if he or she can fall asleep easily at night (in less than 20 minutes), wake easily at his or her normal wake time, and does not require daytime naps (except when developmentally appropriate). ${ }^{6}$ Close to $45 \%$ of high school students were reported to have expressed a need for more sleep on questionnaire surveys. Sleep diaries extending over 1-2 weeks and actigraphy can be used to document sleep length. Management is reported to consist primarily of modifying the daytime schedule in order to obtain more sleep at night. ${ }^{1}$

\section{Circadian rhythm disorders}

This disorder results from the alteration of external cues or the inability to synchronize the internal clock with the external environmental clock. ${ }^{32}$

\section{Delayed sleep phase syndrome}

Delayed sleep phase syndrome is the most common circadian rhythm disorder, accounting for about $10 \%$ of all insomnia complaints. It is related to dysfunction of the suprachiasmatic nucleus, which is the circadian timekeeper. Delayed sleep phase syndrome is associated with inability to advance sleep onset prior to a given time, and the patient is typically a teenager who is unable to fall asleep before 2-3 am. Sleep quality and duration are normal, but sleep onset and offset are at socially inappropriate times, leading to sleep-wake complaints. Treatment therefore consists of keeping a rigid morning wakeup time, and using 1-4 mg of melatonin at bedtime may help induce sleep. Daily exposure to bright light for 30 minutes immediately upon awakening every morning was also recommended because it helps to restore a normal rhythm. ${ }^{1}$ Melatonin has also been used successfully to treat sleep disorders in hyperactive children. In one study, doses of 2.5-5.0 mg nightly provided prompt sedation and improved sleep quality, with no side effects; children have tended to become more alert and sociable, with improvement of their daytime behavior. ${ }^{68}$

\section{Non-24-hour sleep-wake disorder}

Non-24-hour sleep-wake disorder occurs in patients who lack the ability to synchronize their circadian rhythms with the external environment, and continually have a 1-2-hour daily delay in their sleep phases. ${ }^{33}$ The disorder is usually 
observed in blind individuals. Melatonin administration in the evening may be of benefit in this disorder. ${ }^{32}$

\section{Irregular sleep-wake pattern}

Irregular sleep-wake pattern is a total disorganization of the pattern of sleep and wakefulness. The child has normal total sleep time during the 24-hour period, but the periods of sleep and wakefulness occur randomly throughout the day ${ }^{33}$ This disorder is often seen in patients with diffuse brain injury from trauma or perinatal insult. Melatonin administration in the evening may be of benefit in patients with severe brain injury. ${ }^{32}$

\section{Time-zone change syndrome}

Time-zone change syndrome occurs when rapid changes in the day-night cycle outpace the rate at which the suprachiasmatic nuclei can resynchronize to external stimuli, as in the case of air travel. Children tend to respond with less difficulty to these changes than older individuals. ${ }^{32}$ Melatonin may speed up the resynchronization process. ${ }^{69}$

\section{Parasomnias Confusional arousals}

Confusional arousals occur in the first 3 hours after sleep onset when the child experiences an abrupt transition from the deepest phases of NREM sleep, ie, stages 3 and 4, to a lighter stage of sleep. The episodes of arousal are commonly brief in duration, usually lasting $2-10$ minutes. The child often appears confused and disoriented, with incoherent speech, and will re-enter deep sleep and have no recall of the episode next day. ${ }^{6,28,70}$ Predisposing factors for confusional arousals include obstructive sleep apnea, fatigue, sleep deprivation, and irregular wake/sleep schedules, all of which alter stages 3 and 4 NREM sleep. ${ }^{70}$ Prevalence rates are most likely underestimated because children do not recall the events and parents may not observe these night-time events. ${ }^{28}$ Parents should be reassured that these episodes are harmless, will diminish as the child matures, and that they should not try to awaken their child during the episode. ${ }^{6}$

\section{Somnambulism}

In somnambulism (sleep walking) the child sits up in bed with eyes open but is "unseeing." Activity may range from restlessness in bed to walking through the house. The child does not meaningfully interact with people and is often easily agitated; speech is usually mumbled and slurred, and is rarely intelligible. Arousal is difficult and, if successful, the child will appear confused, and recollection of the event is rare. ${ }^{12,15}$ Sleep walking is common, occurring in $1 \%-15 \%$ of children, and usually begins at the age of $4-8$ years, is more common in boys than in girls, and is often associated with enuresis. Because the sleepwalker may try to unlock doors or windows, safety precautions must be taken. Management includes informing parents that the child is truly asleep and should be gently redirected back to bed without awakening. This sleep behavior is usually outgrown by adolescence, although parents should intervene if the child is in danger. Placing a bell on the child's bedroom door to alert parents whenever he leaves the bedroom, and placing locks out of reach of the child are suggested. Bedrooms for sleepwalkers should be on the ground floor of the home, and windows and doors must be secured. ${ }^{6}$ An intervention that proved effective in one study was scheduled awakenings. ${ }^{71}$ Parents kept a diary of the time of sleepwalking over several nights and then began to awaken the child 15 minutes before the sleepwalking had been occurring, making sure the child was fully awake for at least 5 minutes. With the use of this technique, sleepwalking was quickly extinguished in more than $80 \%$ of children.

\section{Somniloquy}

Somniloquy (sleep talking), is another sleep behavior that is usually outgrown by adolescence and does not require any intervention. ${ }^{6,15}$

\section{Sleep terrors}

Sleep terrors (pavor nocturnus) are typified by an extremely agitated child suddenly aroused from stage 4 sleep often screaming, with increased heart rate, and dilated pupils, who appears awake but will not coherently interact with people or surroundings. The child also appears confused and is inconsolable, difficult to arouse, and will not remember the event if aroused or upon awakening in the morning., ${ }^{3,12}$ Sleep terrors are a developmental phenomenon and are not a sign of underlying psychopathology. The disorder is reported in $1 \%-6 \%$ of the pediatric population, generally seen after 18 months of age, and usually disappears by age 6 years. ${ }^{14,28}$ Night terrors occur during stage 3 or 4 NREM sleep, approximately 90 minutes into sleep, so are seen during the first third of the night. It is advised to direct children gently back to their own beds, and they will then promptly fall back to sleep. Children with more violent, prolonged, or unusual episodes should be evaluated at a pediatric sleep disorders center. ${ }^{6}$

\section{Nightmares}

Nightmares are frequently confused with sleep terrors, but there are fundamental differences between the two. The 
average age of onset is 36-72 months, and they occur during REM sleep, so are seen during the last half of the night. Children become fully awake after a nightmare, they can remember graphic details, and frequently remember and talk about their nightmare the next morning. ${ }^{28}$ The incidence of nightmares ranges from $25 \%$ to $50 \%$ in children aged 3-6 years, and both genders are equally affected. ${ }^{12}$ Recurrent nightmares or those with disturbing content may indicate excessive daytime stress. ${ }^{12}$ Clinicians and parents should try to find out the possible cause for stress. Once the cause is revealed, measures should be taken to eliminate the child's exposure to the causative factor. ${ }^{6}$

\section{Nocturnal enuresis}

Nocturnal enuresis is one of the most prevalent sleep problems in children and widely regarded as a parasomnia by most sleep researchers because it occurs only during NREM sleep. The etiology of primary enuresis is likely to be multifactorial. Achieving continence is maturational, and children who lag developmentally at 1 and 3 years of age are more likely to be enuretic at age 6 years. Family history of enuresis is often present. Enuretic children have been reported to have a lower functional bladder capacity, although their true bladder capacity is normal. Other than a thorough history and physical examination with attention to abdominal and neurologic examinations and a urinalysis, more workup is not indicated. Behavioral interventions, such as limiting intake of fluids in the evening and waking the child to use the bathroom before the parent goes to bed have often been tried. Medications, such as imipramine and desmopressin, had been tried as a next step, but multiple studies have demonstrated the superiority of bedwetting alarms in terms of cure rates, lack of side effects, and low relapse rates. ${ }^{15,73}$

\section{Rhythmic movement disorder}

Rhythmic movement disorder involves the rhythmic movement of large muscle groups, such as the head and neck, occurring when the child is attempting to fall asleep, and may continue into stage 1 sleep. Age of onset is usually before the second birthday, and head banging and body rocking are the most common types seen. Polysomnographic monitoring during an episode can rule out seizure disorder. ${ }^{35}$ The disorder is usually transient and self-limited, and rarely requires intervention. $^{72}$

\section{Benign neonatal sleep myoclonus}

Benign neonatal sleep myoclonus is an important parasomnia commonly misdiagnosed as epilepsy. REM sleep in babies is an active period. Along with flickering eye movements, there may be mouth movements and multifocal limb twitches which may cause diagnostic uncertainty, particularly when the jerks are very dramatic. This can be settled by a normal electroencephalogram. The jerks typically occur as the baby is drifting off to sleep, especially at the end of a feeding. They may consist of repetitive, high amplitude, rhythmic myoclonus of arms and legs that continues for seconds to minutes and may not stop simply by gently restraining a limb, but stop if the baby is awakened. When awake, the baby is completely normal. These events gradually disappear during the first 4-6 months of age. Treatment is unnecessary, and benzodiazepines may even exacerbate neonatal sleep myoclonus. ${ }^{57}$

\section{Nocturnal paroxysmal dystonia}

Nocturnal paroxysmal dystonia is a sleep disorder in which movements are often ballistic or choreoathetoid in character and often stereotyped, leading to misdiagnosis as epilepsy. However, prolonged sleep recordings, with extended electroencephalographic montages, fail to document epileptiform activity. They are seen during NREM sleep, and the dystonia may awaken the patient, causing sleep-state disruption. This disorder often responds to carbamazepine. ${ }^{32,33}$

\section{Bruxism}

Bruxism (teeth grinding) primarily occurs in stages I and II or during partial arousals or transitions. No electroencephalographic abnormality is recorded. ${ }^{74}$ It is suggested to result from tension that may originate in unexpressed anger or resentment. Helping the child find ways to express concerns and talking with the child at bedtime to help relieve some of the fears, as well as praise and emotional support, may alleviate the problem. ${ }^{75}$ Bite plates may be needed to prevent dental damage. ${ }^{74}$

\section{Sleep disorders associated with medical disorders}

A wide variety of medical and psychiatric disorders may produce sleep problems, ${ }^{1,33}$ and the following are the most important.

\section{Sleep and epilepsy}

Sleep disturbance is common in epilepsy, and the occurrence of seizures can have profound effects on sleep architecture lasting much longer than the postictal period. Daytime drowsiness in patients with epilepsy is not always due to the side effects of antiepileptics, and may be independently linked with sleep fragmentation. ${ }^{76}$ 
Between $0.5 \%$ and $24 \%$ of people with epilepsy have seizures during sleep. The seizure threshold is often affected by changes in the level of arousal. Many interictal electroencephalographic abnormalities are activated by sleep, and sleep deprivation is well known to lower the seizure threshold. ${ }^{77}$

Landau-Kleffner syndrome is the prototype of epileptic syndromes with electroencephalographic changes primarily augmented during sleep. It is characterized by regression in language, behavioral problems, and infrequent clinical seizures, commonly between three and 10 years of age, in association with epileptiform discharges during both NREM and REM sleep, occurring in more than $80 \%$ of the night sleep epochs, whereby an epoch is 30 seconds of nocturnal polysomnographic tracing. The syndrome of electrical status epilepticus in sleep is similar to Landau-Kleffner syndrome, leading mainly to global neuropsychologic deterioration; the epileptiform features are reported to remain restricted to NREM sleep. Nocturnal frontal lobe epilepsy, another sleeprelated epileptic syndrome, is characterized by brief, 10-15second periods of arousal from stages I and II of NREM sleep that may be accompanied by confusion, thrashing of the limbs, and vocalizations. Nocturnal seizures mimic parasomnias, and video polysomnography is the only definitive test to distinguish between epileptic and nonepileptic events. ${ }^{1}$

Shoukry et a ${ }^{77}$ reported that nocturnal seizures in a child or adolescent are usually linked to benign childhood epilepsies and syndromes because $42.7 \%$ of 96 pediatric epileptic patients with sleep-related seizures and identified epileptic syndromes, had benign childhood epilepsy with centrotemporal spikes, $21.9 \%$ had juvenile myoclonic epilepsy, and $17.4 \%$ had grand mal on awakening epilepsy, $15.6 \%$ had nocturnal frontal lobe epilepsy, and only two patients had electrical status epilepticus in sleep.

It is important to highlight that epileptiform discharges occurring during sleep and their effect on learning and cognitive development have recently gained attention. Many developmental or acquired defects of language or behavior (eg, autism) had been postulated to be a consequence of apparently subclinical epileptiform discharges that interfere with specific cerebral processes, thus impairing plastic changes important for cognitive development and learning. Therefore, it was recommended to suppress these epileptiform discharges. ${ }^{78-84}$

\section{Sleep-related gastroesophageal reflux}

Regurgitation of stomach contents into the esophagus during sleep may lead to aspiration and exacerbate airway disease.
The syndrome may mimic sleep apnea or seizure disorder, and is a major cause of apparent life-threatening events in infants. Diagnosis can be confirmed by radiologic studies of the upper gastrointestinal tract, esophageal $\mathrm{pH}$ probe studies, and in some patients, gastric scintigraphy. Treatment with thickened feeds and placing the child in a more upright position is often helpful. ${ }^{32}$

\section{Sudden infant death syndrome}

Studies focusing on the risk factors for sudden infant death have shown that infants sleeping in the prone position rebreathe exhaled air or have oxygenation problems and decreased arousal ${ }^{85-87}$ Moreover, in this position the pharynx is more collapsible, ${ }^{88}$ thus risking airway obstruction. Genetic alterations in the brain stem, ${ }^{89}$ cell function, ${ }^{90}$ or cytokine profile ${ }^{91}$ were suggested to increase vulnerability in some infants. Cigarette smoke and its causal relationship to sudden infant death syndrome had also been studied, with confirmation of decreased arousal in smoke-exposed infants. ${ }^{92,93}$

\section{Sleep-related asthma}

Children may experience acute episodes of nocturnal dyspnea, arousing them from sleep. Night-time asthma may be due to increased airway resistance that occurs in the early morning. Hormonal changes may play a part in this increase in airway resistance, when cortisol and epinephrine are at their nadir. The decrease in muscle tone during sleep may also increase airway resistance, as well as the decrease in bronchodilator blood levels in the early morning because of increased metabolism. ${ }^{32,74}$ Obstructive sleep apnea may occur concomitantly with asthma; approximately $74 \%$ of asthmatics experience nocturnal symptoms of airflow obstruction secondary to reactive airways disease. Sleep deprivation, chronic upper airway edema, and inflammation associated with obstructive sleep apnea were suggested to exacerbate nocturnal asthma symptoms. Allergic rhinitis may aggravate both obstructive sleep apnea and asthma. Treatment with continuous positive airways pressure therapy has been reported to improve both daytime and night-time peak expiratory flow rates in patients with concomitant obstructive sleep apnea and asthma..$^{51,94}$

\section{Future perspectives}

Finally, it is important to emphasize that sleep problems affect every aspect of the child's development, and that poor sleep in children affects general health, mood, behavior, learning, and higher-level cognitive functions. Moreover, misdiagnosis of sleep disorders often leads to undue investigations 
and excessive medication. Surveys of prevalence of sleep disorders in children, knowledge of parents and health care providers, and their attitude and practice are needed to highlight the scope and the cultural differences in the perception of the problem and improve measures to manage it in the pediatric age group. It is worth mentioning that further studies are needed to elucidate the relationship between subclinical epileptiform discharges during sleep and developmental behavioral disorders, and to identify measures to be taken to prevent the effect of these discharges on brain maturation at this critical period of brain development.

\section{Disclosure}

The author reports no conflicts of interest in this work.

\section{References}

1. Kotagal S. Sleep disturbances. In: Maria BL, editor. Current Management in Child Neurology. 2nd ed. London, UK: BC Decker Inc; 2002 .

2. Chadwick D. Sleep and sleep disorders. In: Donaghy M, editor. Brain's Diseases of the Nervous System. 11th ed. Oxford, UK: Oxford University Press; 2001.

3. Dahl RE. The development and disorders of sleep. Adv Pediatr. 1998;45:73-90.

4. Zee PC, Turek FW. Introduction to sleep and circadian rhythms. In: Turek FW, Zee PC, editors. Regulation of Sleep and Circadian Rhythms. New York, NY: Marcel Dekker Inc; 1999.

5. Davis KF, Parker KP, Montgomery GL. Sleep in infants and young children. Part one: normal sleep. J Pediatr Health Care. 2004; 18(2 Pt 1):65-71.

6. Davis KF, Parker KP, Montgomery GL. Sleep in infants and young children. Part two: Common sleep problems. J Pediatr Health Care. 2004;18(3 Pt 2):130-137.

7. Simon R, Sunseri M. Disorders of sleep and arousal. In: Goldman L, Ausiello D, editors. Cecil Textbook of Medicine. 22nd ed. Philadelphia, PA: Saunders; 2004.

8. Czeisler CA, Winkelman JW, Richardson GS. Sleep disorders. In: Braunwald E, Fauci A, Kasper D, Hauser S, Longo D, Jameson J, editors. Harrison's Principles of Internal Medicine. 15th ed. New York, NY: McGraw-Hill; 2001.

9. Davis FC, Heller HC, Frank MG. Ontogeny of sleep and circadian rhythms. In: Turek FW, Zee PC, editors. Regulation of Sleep and Circadian Rhythms. New York, NY: Marcel Dekker Inc; 2000.

10. Mistlberger RE, Rusak B. Circadian rhythms in mammals: Formal properties and environmental influences. In: Kryger MH, Roth $\mathrm{T}$, Dement WC, editors. Principles and Practice Of Sleep Medicine. 3rd ed. Philadelphia, PA: WB Saunders; 2000.

11. Anders TF, Sadeh A, Appareddy V. Normal sleep in neonates and children. In: Ferber R, Kryger M, editors. Principles and Practice of Sleep Medicine in the Child. 3rd ed. Philadelphia, PA: WB Saunders; 1995.

12. Adair RH, Bauchner H. Sleep problems in childhood. Curr Probl Pediatr. 1993;23:147-170.

13. Sheldon SH. Sleep in infants and children. In: Lee-Chiong TL, Sateia MJ, Carskadon MA, editors. Sleep Medicine. Philadelphia, PA: Hanley and Belfus Inc; 2002.

14. Anders TF, Eiben LA. Pediatric sleep disorders. A review of the past 10 years. J Am Acad Child Adolesc Psychiatry. 1997;36:9-20.

15. Thiedke C. Sleep disorders and sleep problems. Am Fam Physician. 2001;63:277-284.
16. Owens JA, Spirito A, McGuinn M, Nobile C. Sleep habits and sleep disturbance in elementary school-aged children. $J$ Dev Behav Pediatr. 2000;21:27-36.

17. Ohayon MM, Roberts FE, Zulley J, Smirne S, Priest RG. Prevalence and patterns of problematic sleep among older adolescents. J Am Acad Child Adolesc Psychiatry. 2000;39:1549-1556.

18. Carskadon MA, Wolfson AR, Acebo C, Tzischinsky O, Seifer R. Adolescent sleep patterns, circadian timing, and sleepiness at a transition to early school days. Sleep. 1998;21:871-881.

19. Wolfson AR, Carskadon MA. Sleep schedules and daytime functioning in adolescents. Child Dev. 1998;69:875-897.

20. Johnson CR. Sleep problems in children with mental retardation and autism. Child Adolesc Psychiatr Clin North Am. 1996;5:673-680.

21. Mindell J, Owens J. Sleep problems in pediatric practice. Clinical issues for the pediatric nurse practitioner. J Pediatr Health Care. 2003;17: 324-331.

22. Fallone G, Owens JA, Deane J. Sleepiness in children and adolescents: Clinical implications. Sleep Med Rev. 2002;6:287-306.

23. Gregory AM, Eley TC, O'Connor TG, Plomin R. Etiologies of associations between childhood sleep and behavioural problems in a large twin sample. J Am Acad Child Adolesc Psychiatry. 2004;43:744-751.

24. Scheen AJ, Van Cauter E. The roles of time of day and sleep quality in modulating glucose regulation: Clinical implications. Horm Res. 1998;49:191-201.

25. Spiegel K, Leprout R, Van Cauter E. Impact of sleep debt on metabolic function. Lancet. 1999;354:1435-1439.

26. Stores G. Children's sleep disorders: Modern approaches, developmental effects, and children at risk. Dev Med Child Neurol. 1999;41:568-573.

27. Wiggs L, Stores G. Behavioural treatment for sleep problems in children with severe intellectual disabilities and daytime challenging behavior: Effects on mothers and fathers. Br J Health Psychol. 2001;6:257-269.

28. Mindell J, Owens J, Carskadon M. Developmental features of sleep. Child Adolesc Psychiatr Clin North Am. 1999;8:695-725.

29. Owens JA. Challenges in managing sleep problems in young children. West J Med. 2000;173:38.

30. Willinger M, Ko C-W, Hoffman HJ, Kessler R, Corwin MJ. Trends in infant bed sharing in the United States, 1993-2000. Arch Pediatr Adolesc Med. 2003;157:43-49.

31. Meissner HH, Riemer A, Santiago SM, Stein M, Goldman MD, Williams AJ. Failure of physician documentation of sleep complaints in hospitalized patients. West J Med. 1998;169:146-149.

32. Kohrman M. Pediatric sleep disorders. In: Swaiman K, Ashwal S, editors. Pediatric Neurology Principles and Practice. 3rd ed. St Louis, MO: Mosby; 1999.

33. American Sleep Disorders Association. The International Classification of Sleep Disorders: Diagnostic and Coding Manual. Rochester, MN: American Sleep Disorders Association; 1997.

34. American Academy of Sleep Medicine. The International Classification of Sleep Disorders, Revised: Diagnostic and Coding Manual. Westchester, IL: American Academy of Sleep Medicine; 2001. Available from: http://www.esst.org/adds/ICSD.pdf. 2001. Accessed July 15, 2011.

35. Halbower A, Marcus C. Sleep disorders in children. Curr Opin Pulm Med. 2003;9:471-476.

36. American Psychiatric Association. Diagnostic and Statistical Manual of Mental Disorders Fourth Edition Text Revision. Washington, DC: American Psychiatric Association; 2000.

37. Lewis KL. Apneas, hypopneas, and respiratory effort-related arousals: Moving closer to a standard. Curr Opin Pulm Med. 2002;8:493-497.

38. Marcus CL. Sleep-disordered breathing in children. Am J Respir Crit Care Med. 2001;164:16-30.

39. Sheldon SH. Polysomnography in infants and children. In: Sheldon SH, Ferber R, Kryger MH, editors. Principles and Practice of Pediatric Sleep Medicine. Philadelphia, PA: Elsevier Saunders; 2005.

40. Merritt S. Sleep-disordered breathing and the association with cardiovascular risk. Prog Cardiovasc Nurs. 2004;19:19-27. 
41. Baldwin CM, Quan SF. Sleep disordered breathing. Nurs Clin North Am. 2002;37:633-654.

42. Chan J, Edman J, Koltai P. Obstructive sleep apnea in children. Am Fam Physician. 2004;1147-1160.

43. Blunden S, Lushington K, Kennedy D, Martin J, Dawson D. Behavior and neurocognitive performance in children aged 5-10 years who snore compared to controls. J Clin Exp Neuropsychol. 2000;22:554-568.

44. Gozal D, Pope DW Jr. Snoring during early childhood and academic performance at ages thirteen to fourteen years. Pediatrics. 2001;107:1394-1399.

45. Gozal D, Wang M, Pope DW Jr. Objective sleepiness measures in pediatric obstructive sleep apnea. Pediatrics. 2001;108:693-697.

46. Freezer NJ, Bucens IK, Robertson CF. Obstructive sleep apnoea presenting as failure to thrive in infancy. J Paediatr Child Health. 1995;31:172-175.

47. Umlauf MG, Chasens ER. Sleep disordered breathing and nocturnal polyuria: Nocturia and enuresis. Sleep Med Rev. 2003;7:403-411.

48. Montgomery-Downs HE, O'Brien LM, Gulliver TE, Gozal D. Polysomnographic characteristics in normal preschool and early schoolaged children. Pediatrics. 2006;117:741-753.

49. Ariagno RL, Mirmiran M, Adams MM, Saporito AG, Dubin AM, Baldwin RB. Effect of position on sleep, heart rate variability, and QT interval in preterm infants at 1 and 3 months' corrected age. Pediatrics 2003;111:622-625.

50. Marcus CL, Ward SL, Mallory GB, et al. Use of nasal continuous positive airway pressure as treatment of childhood obstructive sleep apnea. J Pediatr. 1995;127:88-94.

51. Qureshi A, Ballard RD. Obstructive sleep apnea. J Allergy Clin Immunol. 2003;112:643-652.

52. Al-Ghamdi SA, Manoukian JJ, Morielli A, Oudjhane K, Ducharme FM, Brouillette RT. Do systemic corticosteroids effectively treat obstructive sleep apnea secondary to adenotonsillar hypertrophy. Laryngoscope. 1997; 107:1382-1387.

53. Brouillette RT, Manoukian JJ, Ducharme FM, et al. Efficacy of fluticasone nasal spray for pediatric obstructive sleep apnea. J Pediatr. 2001;138:838-844.

54. Rosen CL, Haddad GG. Obstructive sleep apnea and hypoventilation in children. In: Behrman R, Kliegman R, Jenson H, editors. Nelson Textbook of Pediatrics. 16th ed. Philadelphia, PA: WB Saunders; 2000.

55. Hoeve HL, Joosten KF, Van Den Berg S. Management of obstructive sleep apnea syndrome in children with craniofacial malformation. Int J Pediatr Otorhinolaryngol. 1999;49 Suppl 1:S59-S61.

56. Wise MS, Lynch J. Narcolepsy in children. Semin Pediatr Neurol. 2001;8:198-206.

57. Camfield P, Camfield C. Nonepileptic events simulating seizures in childhood. In: Maria BL, editor. Current Management In Child Neurology. 2nd ed. London, UK: BC Decker Inc; 2002.

58. Guilleminault C, Pelayo R. Narcolepsy in children: A practical guide to its diagnosis, treatment and follow-up. Paediatr Drugs. 2000;2:1-9.

59. Tsukamoto H, Ishikawa T, Fujii Y, Fukumizu M, Sugai K, Kanbayashi T. Undetectable levels of CSF hypocretin-1 (OREXIN-A) in two prepubertal boys with narcolepsy. Neuropediatrics. 2002;33:51-52.

60. Krahn L. Sleep disorders. Semin Neurol. 2003;23:307-314.

61. Scharf M, Lai A, Branigan B, Stover R, Berkowitz D. Pharmacokinetics of gammahydroxybutyrate (GHB) in narcoleptic patients. Sleep. 1998;21: 507-514.

62. Zyrem Multicenter Study Group. A randomized, double blind, placebocontrolled multicenter trial comparing the effects of three doses of orally administered sodium oxybate with placebo for the treatment of narcolepsy. Sleep. 2002;25:42-49.

63. Dauvilliers Y, Mayer G, Lecendreux M, et al. Kleine-Levin syndrome: An autoimmune hypothesis based on clinical and genetic analyses. Neurology. 2002;59:1739-1745.

64. Ferber R. Childhood sleep disorders. Neurol Clin. 1996;14:493-511.

65. Mindell J. Empirically supported treatments in pediatric psychology: Bedtime refusal and night wakings in young children. J Pediatr Psychol. 1999;24:465-481.
66. Lee-Chiong TL. Manifestations and classification of sleep disorders. In: Lee-Chiong T1, Sateia MJ, Carskadon MA, editors. Sleep Medicine. Philadelphia, PA: Hanley and Belfus Inc; 2002.

67. Dahl RE. Sleep in behavioral and emotional disorders. In: Ferber R, Kryger M, editors. Principles and Practice Of Sleep Medicine in the Child. 3rd ed. Philadelphia, PA: WB Saunders; 1995.

68. Barclay L. Melatonin helpful for delayed sleep onset in children with ADHD. Abstract E16, presented at the American Academy Of Child And Adolescent Psychiatry 50th annual meeting, October 14-19, 2003, Miami, FL.

69. Sack R, Blood M, Lewy A. Phase shifts in melatonin rhythms are highly variable among night shift workers on the same schedule. Sleep Res. 1994:23:78.

70. Rosen G, Mahowald MW, Ferber R. Sleepwalking, confusional arousals, and sleep terrors in the child. In: Ferber R, Kryger M, editors. Principles and Practice of Sleep Medicine in the Child. Philadelphia, PA: WB Saunders: 1995.

71. Frank NC, Spirito A, Stark L, Owens-Stively J. The use of scheduled awakenings to eliminate childhood sleepwalking. J Pediatr Psychol. 1997;22:345-353.

72. Hoban TF. Rhythmic movement disorder in children. CNS Spectr. $2003 ; 8: 135-138$.

73. Wan J, Greenfield S. Enuresis and common voiding abnormalities. Pediatr Clin North Am. 1997;44:1117-1131.

74. Sadock B, Sadock V. Sleep disorders. In: Sadock B, Sadock V, editors. Kaplan and Sadock's Pocket Handbook of Clinical Psychiatry. 3rd ed. Philadelphia, PA: Lippincott, Williams and Wilkins; 2002.

75. Dalton R. Habit disorders. In: Behrman R, Kliegman R, Jenson H, editors. Nelson Textbook of Pediatrics. 16th ed. Philadelphia, PA: WB Saunders; 2000.

76. Bazil CW. Epilepsy and sleep disturbance. Epilepsy Behav. 2003;4:S39-S45.

77. Shoukry I, Azmy A, El Shakankiry H, El Sebaie, Gamal N. Childhood sleep related seizures. Epilepsia. 2003;44 Suppl 9:Abstr 2379.

78. El Shakankiry H. Epileptiform discharges augmented during sleep: Is it a trait with diverse clinical presentation according to age of expression? Epilepsy Res. 2010;89:113-120.

79. Eriksson K, Kylliainen A, Hirvonen K, Nieminen P, Koivikko M. Visual agnosia in a child with non-lesional occipito-temporal CSWS. Brain Dev. 2003;25:262-267.

80. Guerrini R, Arzimanoglou A, Rrouwer O. Rationale for treating epilepsy in children. Epileptic Disord. 2002:S9-S21.

81. Laporte N, Sebire G, Gillerot Y, Guerrini R, Ghariani S. Cognitive epilepsy ADHD related to focal EEG discharges. Pediatr Neurol. 2002;27:307-311.

82. Pressler RM, Robinson RO, Wilson GA, Binnie CD. Treatment of interictal epileptiform discharges can improve behavior in children with behavioral problems and epilepsy. J Pediatr. 2005;146: $112-117$.

83. Tassinari CA, Rubboli G, Volpi L, Billard C, Bureau M. Encephalopathy with electrical status epilepticus during slow sleep or ESES syndrome including the acquired aphasia. Clin Neurophysiol. 2000;111 Suppl 2: S94-S102.

84. Tassinari CA, Rubboli G, Volpi L, Billard C, Bureau M. Electrical status epilepticus during slow sleep (ESES or CSWS) including acquired aphasia (Landau-Kleffner syndrome). In: Roger J, Bureau M, Dravet C, Genton P, Tassinari CA, Wolf P, editors. Epileptic Syndromes in Infancy, Childhood And Adolescence. 4th ed. London, UK: John Libbey Eurotext; 2005.

85. Horne RS, Ferens D, Watts AM, et al. The prone sleeping position impairs arousability in term infants. J Pediatr. 2001;138:811-816.

86. Horne RS, Bandopadhayay P, Vitkovic J, Cranage SM, Adamson TM. Effects of age and sleeping position on arousal from sleep in preterm infants. Sleep. 2002;25:746-750.

87. Patel AL, Paluszynska D, Harris KA, Thach BT. Occurrence and mechanisms of sudden oxygen desaturation in infants who sleep face down. Pediatrics. 2003;111(4 Pt 1):E328-E332. 
88. Ishikawa T, Isono S, Aiba J, Atsuko T, Takashi N. Prone position increases collapsibility of the passive pharynx in infants and small children. Am J Respir Crit Care Med. 2002;166:760-764.

89. Kinney HC, Filiano JJ, White WF. Medullary serotonergic network deficiency in the sudden infant death syndrome: Review of a 15-year study of a single dataset. J Neuropathol Exp Neurol. 2001;60:228-247.

90. Ackerman MJ, Siu BL, Sturner WQ, et al. Postmortem molecular analysis of SCN5A defects in sudden infant death syndrome. JAMA. 2001;286:2264-2269.
91. Gordon AE, Mackenzie DA, El Ahmer OR, et al. Evidence for a genetic component in sudden infant death syndrome. Child Care Health Dev. 2002;28 Suppl 1:27-29.

92. Nattie E, Kinney H. Nicotine, serotonin, and sudden infant death syndrome. Am J Respir Crit Care Med. 2002;166:1530-1531.

93. Chang AB, Wilson SJ, Masters IB, et al. Altered arousal response in infants exposed to cigarette smoke. Arch Dis Child. 2003;88:30-33.

94. Bonekat HW, Hardin KA. Severe upper airway obstruction during sleep. Clin Clin Rev Allergy Immunol. 2003;25:191-210.

\section{Publish your work in this journal}

Nature and Science of Sleep is an international, peer-reviewed, open access journal covering all aspects of sleep science and sleep medicine, including the neurophysiology and functions of sleep, the genetics of sleep, sleep and society, biological rhythms, dreaming, sleep disorders and therapy, and strategies to optimize healthy sleep. The journal welcomes

\section{Dovepress}

original research, clinical \& epidemiological studies, reviews \& evaluations, case reports and extended reports. The manuscript management system is completely online and includes a very quick and fair peerreview system, which is all easy to use. Visit http://www.dovepress.com/ testimonials.php to read real quotes from published authors. 